\title{
Synchrotron X-ray diagnostics of cutoff shape of nonthermal electron spectrum at young supernova remnants
}

\author{
Ryo Yamazaki ${ }^{1}$, Yutaka Ohira ${ }^{1}$, Makoto Sawada ${ }^{1}$, and Aya Bamba ${ }^{1}$ \\ Department of Physics and Mathematics, Aoyama Gakuin University, 5-10-1 Fuchinobe, Sagamihara \\ 252-5258, Japan; ryo@phys.aoyama.ac.jp
}

\begin{abstract}
Synchrotron X-rays can be a useful tool to investigate electron acceleration at young supernova remnants (SNRs). At present, since the magnetic field configuration around the shocks of SNRs is uncertain, it is not clear whether electron acceleration is limited by SNR age, synchrotron cooling, or even escape from the acceleration region. We study whether the acceleration mechanism can be constrained by the cutoff shape of the electron spectrum around the maximum energy. We derive analytical formulae of the cutoff shape in each case where the maximum electron energy is determined by SNR age, synchrotron cooling and escape from the shock. They are related to the energy dependence of the electron diffusion coefficient. Next, we discuss whether information on the cutoff shape can be provided by observations in the near future which will simply give the photon indices and the flux ratios in the soft and hard X-ray bands. We find that if the power-law index of the electron spectrum is independently determined by other observations, then we can constrain the cutoff shape by comparing theoretical predictions of the photon indices and/or the flux ratios with observed data which will be measured by NUSTAR and/or ASTRO-H. Such study is helpful in understanding the acceleration mechanism. In particular, it will supply another independent constraint on the magnetic field strength around the shocks of SNRs.
\end{abstract}

Key words: Acceleration of particles - ISM: cosmic rays - ISM: supernova remnants Turbulence - X-rays: ISM

\section{INTRODUCTION}

Observations of synchrotron X-rays indicate that young supernova remnants (SNRs) act as electron accelerator up to 10-100 TeV (e.g., Koyama et al. 1995). Spectral fitting of the synchrotron radiation from radio to X-ray bands gives us information on the acceleration mechanism. For example, the maximum energy of electrons $E_{\max , \mathrm{e}}$ and the magnetic field strength $B$ are constrained by measuring the roll-off frequency of the spectrum, $\nu_{\text {roll }}$, which is proportional to $B E_{\max , e}{ }^{2}$ (Reynolds 1998, Reynolds $\&$ Keohane 1999). Furthermore, if the rapid cooling time of $1-10 \mathrm{yr}$ is responsible for the narrowness of thin filaments close to the shock front (Vink \& Laming 2003, Bamba et al. 2003, 2005a, 2005b, Yamazaki et al. 2004) and/or time variability of synchrotron X-rays (Uchiyama et al. 2007, Uchiyama \& Aharonian 2008), then $B \sim 0.1-1 \mathrm{mG}$ is inferred, so that $E_{\text {max,e }} \sim 10 \mathrm{TeV}$. Given the values of $E_{\mathrm{max}, \mathrm{e}}$ and $B$ as well as the SNR age $t_{\mathrm{age}}$, we can even estimate the maximum energy of protons $E_{\text {max }, \mathrm{p}}$, which is proportional to $E_{\max , \mathrm{e}}{ }^{2} B^{2} t_{\text {age }}$ (see Section A.11.

For young SNRs, $\nu_{\text {roll }}$ is typically below the X-ray band (e.g., Bamba et al. 2003, 2005a, 2005b), so that synchrotron X-rays are emitted by electrons whose energy is near $E_{\text {max,e. }}$. Hence it is expected that information on the electron spectrum at highest energy range can be extracted from the soft and hard 
X-ray data. In particular, the recently launched satellite The Nuclear Spectroscopic Telescope Array (NuSTAR) (Hailey et al.2010, Harrison et al.2013) and ASTRO-H (Takahashi et al.2010) that will start in the near future will observe hard X-rays whose photon energy is larger than $10 \mathrm{keV}$. In this paper, we focus on the cutoff shape of the electron spectrum and show that it may provide another independent way to tackle the problems of particle acceleration at young SNRs (Section 2). Then, we show that observations by these satellites will play an important role in this context (Section 3 and 4).

\section{CUTOFF SHAPE OF THE ELECTRON SPECTRUM}

We assume the shape of the electron spectrum around the maximum energy, $E_{\mathrm{max}, \mathrm{e}}$, in the simple form,

$$
N(E) \propto E^{-p} \exp \left[-\left(E / E_{\max , \mathrm{e}}\right)^{a}\right] .
$$

As discussed in the following, the spectral index $p$ and the cutoff shape parameter $a$ contain rich information on the acceleration mechanism. We parameterize the functional form of the electron spectrum, Equation (1), to be as simple as possible so that the result can be compared with observations. In practice, when we consider X-ray synchrotron emission from typical young SNRs, only the energy range around $E_{\max , \mathrm{e}}$ is important because the roll-off energy of the synchrotron radiation is much smaller than $2 \mathrm{keV}$, so that detailed fluctuations of the electron spectrum caused by more realistic but somewhat complicated models, such as the nonlinear model, may not be important.

\subsection{Spectral index $p$}

When the diffusive shock acceleration works at the adiabatic shock with a compression ratio of $r$ and the energy loss effects are negligible, the spectral index is given by $p=(r+2) /(r-1)$ in the test-particle limit (Bell 1978, Blandford \& Ostriker 1978). In particular, $p=2.0$ in the case of the strong shock limit $r=4$. This simplest case may not be applicable for actual young SNRs, in which GeV-to-TeV gamma-ray observations of youngest SNRs such as Cas A and Tycho suggest $p>2.0$ (Abdo et al.2010, Giordano et al. 2012). This fact has already been inferred from both the radio synchrotron spectrum and the propagation model of cosmic rays (e.g., Strong \& Moskalenko 1998, Putze et al. 2009; Shibata et al.2011).

If the magnetic field is strong enough (e.g., in the Bohm diffusion model, $K(E) \propto E$, see equation (A.5), synchrotron cooling is responsible for a spectral break, above which the electron spectrum becomes softer and the spectral index increases by 1.0, so that $p>3.0$ (e.g., Longair 1994). Note that the spectral steepening by one power occurs for homogeneous stationary sources. It is also noted that sometimes the synchrotron cooling effect causes spectral hardening or pile-up (Longair 1994; Drury et al. 1999, Zirakashvili \& Aharonian 2007). For typical parameters of young SNRs, however, such a hard component can hardly be seen for more than a decade of electron energy. At most a small bump is formed just below $E_{\mathrm{max}, \mathrm{e}}$, which may be seen as a small excess in the radiation spectrum (see Section 4).

\subsection{Cutoff shape parameter $a$}

The cutoff shape parameter $a$ also depends on the details of electron acceleration, such as the magnetic field strength and the energy dependence of the diffusion coefficient. We assume that the diffusion coefficient of high-energy electrons has the following power-law form

$$
K(E) \propto E^{\beta} .
$$

Usually the Bohm diffusion, $\beta=1$, is widely adopted. However, in general $\beta$ may deviate from unity. For example, it is well known that $\beta$ becomes $1 / 3$ if particle diffusion is considered in the Kolmogorov magnetic turbulence, but $\beta=1 / 2$ for Kraichnan turbulence (e.g., Blandford \& Eichler 19871]. So in

\footnotetext{
1 In general, if the spectrum of magnetic turbulence has a form $E_{k} \propto k^{-s}$, then $\beta$ and $s$ are related as $\beta=2-s$ under the assumption that accelerated particles scatter via wave-particle resonance interaction. In particular, for Kolmogorov $(s=5 / 3)$ and Kraichnan $(s=3 / 2)$ turbulence, we obtain $\beta=1 / 3$ and $1 / 2$, respectively.
} 
these cases, the value of $\beta$ even tells us the properties of magnetic turbulence. In another context, if the wave damping due to ion-neutral collisions is significant, $\beta$ may approach $\approx 2$ (Bykov et al. 2000, Lee et al.2012).

In the cooling limited case, where $E_{\mathrm{max}, \mathrm{e}}$ is determined by the balance of acceleration and synchrotron cooling $\left(t_{\mathrm{acc}}(E)=t_{\mathrm{syn}}(E)\right)$, the spectral shape factor $a$ is related to $\beta$ as

$$
a=\beta+1,
$$

which is analytically derived as in Section B.1. In the age-limited case, where the synchrotron cooling effect is neglected and $E_{\max , \mathrm{e}}$ is determined by the finite age, Kang et al. (2009) have given

$$
a=2 \beta,
$$

by fitting their results of numerical simulation (see also Kato \& Takahara 2003). Note that in the case of Bohm diffusion (that is, $\beta=1$ ), both age-limited and cooling-limited cases give the same value, $a=2$. If $\beta \neq 1$, the values of $a$ for the two cases are different.

It may happen that the maximum energy is limited by the escape process (Ptuskin \& Zirakashvili 2005, Drury et al. 2009, Caprioli et al. 2009, Ohira et al. 2010). Here we consider the simplest case (see Section B.2), in which a free escape boundary exists upstream of the shock front. In the test particle limit, we analytically derive

$$
a=\beta .
$$

If nonlinear effects, in particular the decay of self-excited upstream turbulence, are taken into account, then $a$ may be slightly larger (e.g., Lee et al.2012) although the precise cutoff shape is at present highly uncertain (Ellison \& Bykov 2011). However, as long as $\beta \approx 1$, the escape process does not affect the maximum electron energy (Ohira et al.2012b) and hence the cutoff shape for young SNRs.

Equations (3), (4), and (5), are derived on the assumption that the shock velocity is constant with time, but the shock velocity of real SNRs decreases with time after the free expansion phase. Some young SNRs are still in the free expansion phase or have just entered the Sedov phase, so that the effect of shock deceleration is not important. Even if the shock velocity is decreasing, our assumption that the shock is stationary may not significantly influence the result because of the following reasons. In the cooling limited and escape limited cases, the acceleration time of electrons with arbitrary energy is smaller than the dynamical time, so that the shock velocity can be treated as stationary. Even in the age-limited case, high-energy particles produced around the shock suffer adiabatic expansion after they are transported downstream of the shock and lose their energy. Hence, at a given epoch, the spectrum for high-energy particles is dominated by those which are being accelerated at that time, in other words, the energy spectrum of particles does not depend so much on the past acceleration history. In particular, we are now interested in the energy region near the upper end of the spectrum because as seen in the following, X-rays are produced by particles with energy near the maximum energy. In this energy regime, the electron spectrum is well approximated by that for the stationary shock case.

So far, we have discussed the cutoff shape in the diffusion approximation for particle motion. For the cooling limited and the age limited cases, the diffusion approximation is valid even around the maximum energy, because their gyro radii are much shorter than the size of the acceleration region and/or escape boundary. For the escape-limited case, the mean free path of a particle becomes large around the escape boundary, so that the diffusion approximation is invalid around the escape boundary and the cutoff shape

could be modified. Even so, it can be said that the cutoff shape contains rich information on the particle acceleration.

\section{SYNCHROTRON X-RAY DIAGNOSTICS OF CUTOFF SHAPE}

In this section, we consider the synchrotron radiation from electrons whose energy distribution is given by equation (1). Assuming an isotropic pitch-angle distribution of electrons, the energy spectrum $F_{\nu}$ [erg s ${ }^{-1} \mathrm{~cm}^{-2} \mathrm{~Hz}^{-1}$ ] of the synchrotron radiation is computed. The energy flux, in the photon 
energy range between $\varepsilon_{1}=h \nu_{1}$ and $\varepsilon_{2}=h \nu_{2}\left(\nu_{1}<\nu_{2}\right)$, is given by $F\left(\varepsilon_{1}-\varepsilon_{2}\right)=\int_{\nu_{1}}^{\nu_{2}} F_{\nu} d \nu$. We calculate photon indices and flux ratios in various energy bands, in which we focus in the following. These spectral quantities are determined if we specify four parameters, $a, p, E_{\max , \mathrm{e}}$ and the magnetic field strength $B$ in the emitting region. Note that the flux normalization is not necessary. The field strength $B$ is only required to determine the frequency that gives the peak of the $\nu F_{\nu}$ for the synchrotron radiation, a so called roll-off frequency $\nu_{\text {roll }}\left(\propto B E_{\text {max }, e^{2}}\right)$, which roughly corresponds to the characteristic synchrotron frequency of electrons with $E_{\max , \mathrm{e}}$ (Reynolds 1998, Reynolds \& Keohane 1999). Different parameter sets but with the same value of $B E_{\max , \mathrm{e}^{2}}$ give the same photon indices and flux ratios. Therefore, independent parameters are $a, p$ and $B E_{\max , \mathrm{e}^{2}}$.

Figure 1 shows the $10-50 \mathrm{keV}$ photon index as a function of 2-10 keV photon index. We adopt $p=2.0$ (thick lines) and 3.0 (thin lines), and $a=0.5$ (light blue), 1 (green), 2 (red), 3 (blue) and 4 (purple). Along each line, both $a$ and $p$ are constant, and the quantity $B E_{\max , \mathrm{e}}{ }^{2}$ changes. In the limiting case of $B E_{\max , \mathrm{e}^{2}} \rightarrow \infty$, rolloff frequency $\nu_{\text {roll }}$ goes beyond the observation band, which implies the synchrotron spectrum is well approximated by $F_{\nu} \propto \nu^{-(p-1) / 2}$. Then the photon index becomes an asymptotic value,

$$
\Gamma_{\infty}=\frac{p+1}{2},
$$

so that $\Gamma_{\infty}=1.5$ and 2.0 for $p=2.0$ and 3.0, respectively, which correspond to the left end of each line. When $B E_{\max , \mathrm{e}^{2}}$ decreases, the rolloff frequency $\nu_{\text {roll }}$ crosses the observation bands, so the photon indices become larger than the asymptotic value $\Gamma_{\infty}$. After passing through the harder band $10-50 \mathrm{keV}$, $\nu_{\text {roll }}$ crosses the softer 2-10 keV band, so the photon index in the former band is larger than the latter one. For fixed $p$, if $a$ becomes larger, the flux beyond $\nu_{\text {roll }}$ decreases more rapidly, resulting in a larger photon index. Hence each line has a steeper slope for larger $a$. The value of $B E_{\max , \mathrm{e}}{ }^{2}$ is reduced to $10^{3} \mu \mathrm{G}(\mathrm{TeV})^{2}$, which corresponds to the characteristic frequency of the synchrotron radiation of $2.9 \times 10^{16} \mathrm{~Hz}$. Light blue lines in all the Figures as well as the thick green line in Figure 4 have a right end, which corresponds to this lower limit.

Figure 2 shows the flux ratio $R_{1}=F(10-30 \mathrm{keV}) / F(30-80 \mathrm{keV})$ as a function of the $2-10 \mathrm{keV}$ photon index. In the case of $B E_{\max , \mathrm{e}}{ }^{2} \rightarrow \infty$, the flux ratio $R=F\left(\varepsilon_{1}-\varepsilon_{2}\right) / F\left(\varepsilon_{3}-\varepsilon_{4}\right)$ of two energy bands $\varepsilon_{1}-\varepsilon_{2}[\mathrm{keV}]$ and $\varepsilon_{3}-\varepsilon_{4}[\mathrm{keV}]$ has an asymptotic value

$$
R \rightarrow \frac{\varepsilon_{2}^{2-\Gamma_{\infty}}-\varepsilon_{1}^{2-\Gamma_{\infty}}}{\varepsilon_{4}^{2-\Gamma_{\infty}}-\varepsilon_{3}^{2-\Gamma_{\infty}}},
$$

for $\Gamma_{\infty} \neq 2$, but

$$
R \rightarrow \frac{\ln \left(\varepsilon_{2} / \varepsilon_{1}\right)}{\ln \left(\varepsilon_{4} / \varepsilon_{3}\right)},
$$

for $\Gamma_{\infty}=2$. Hence in the present case $\left(\varepsilon_{1}=10 \mathrm{keV}, \varepsilon_{2}=\varepsilon_{3}=30 \mathrm{keV}\right.$ and $\left.\varepsilon_{4}=80 \mathrm{keV}\right)$, we have $\Gamma_{\infty}=1.5$ and $R_{1} \rightarrow 0.668$ for $p=2.0$, but $\Gamma_{\infty}=2.0$ and $R_{1} \rightarrow 1.12$ for $p=3.0$. When $B E_{\text {max }, \mathrm{e}}{ }^{2}$ becomes small, both the flux ratio and the $2-10 \mathrm{keV}$ photon index become large, however the decay slope is steeper for large $a$.

Figure 3 shows the flux ratio $R_{2}=F(2-10 \mathrm{keV}) / F(10-80 \mathrm{keV})$ as a function of the $2-10 \mathrm{keV}$ photon index. In the limit of $B E_{\text {max }, \mathrm{e}}{ }^{2} \rightarrow \infty$, we have $\Gamma_{\infty}=1.5$ and $R_{2} \rightarrow 0.302$ for $p=2.0$, but $\Gamma_{\infty}=2.0$ and $R_{2} \rightarrow 0.774$ for $p=3.0$.

Figure 4 shows the flux ratio $R_{1}=F(10-30 \mathrm{keV}) / F(30-80 \mathrm{keV})$ as a function of $R_{3}=$ $F(2-10 \mathrm{keV}) / F(10-30 \mathrm{keV})$. In the limit of $B E_{\max , \mathrm{e}}{ }^{2} \rightarrow \infty$, we have $R_{1} \rightarrow 0.668$ and $R_{3} \rightarrow 0.755$ for $p=2.0$, but $R_{1} \rightarrow 1.12$ and $R_{3} \rightarrow 1.46$ for $p=3.0$.

Figures 5 and 6 are the same as Figs. 1 and 2 respectively, but for $p=2.3$ and 3.3. The former is typical for the source spectrum of Galactic cosmic rays, which is inferred by the propagation model (e.g., Strong \& Moskalenko 1998, Putze et al. 2009, Shibata et al.2011). It is also expected from gamma-ray observations that young SNRs such as Cas A and Tycho have an energy spectrum with $p=2.3$ (see Section 4.1.2. Abdo et al. 2010). The latter case $(p=3.3)$ is realized if the synchrotron cooling is 


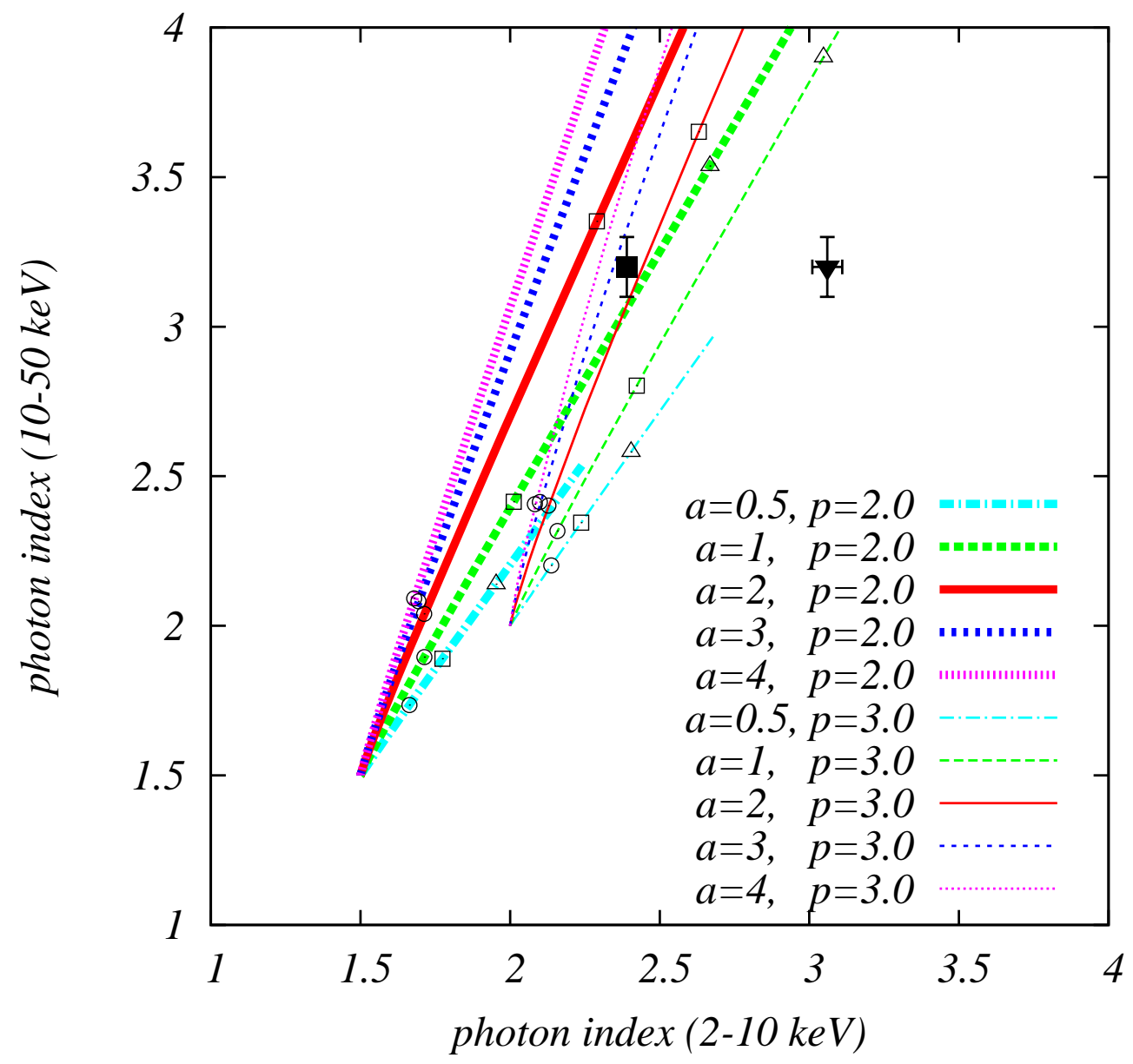

Fig. 1 Photon index in the $10-50 \mathrm{keV}$ band as a function of the $2-10 \mathrm{keV}$ photon index. Along each line, both $a$ and $p$ are constant, and the quantity $B E_{\max , \mathrm{e}^{2}}$ changes from $10^{3} \mu \mathrm{G}(\mathrm{TeV})^{2}$ to $\infty$; open triangles, squares, and circles are for $B E_{\max , \mathrm{e}^{2}}=10^{4}, 10^{5}$ and $10^{6} \mu \mathrm{G}(\mathrm{TeV})^{2}$, respectively. Thick lines are for $p=2.0$, while thin lines are for $p=3.0$. Light blue, green, red, blue and purple lines are for $a=0.5,1,2,3$ and 4, respectively. The filled square and triangle are observed data for RX J1713.7-3946 and Cas A, respectively (see section 4.1).

significant enough to provide a cooling break below which the electron spectrum has $p=2.3$. In the limit of $B E_{\max , \mathrm{e}^{2}} \rightarrow \infty$, we have $\Gamma_{\infty}=1.65$ and $R_{1} \rightarrow 0.779$ for $p=2.3$, but $\Gamma_{\infty}=2.15$ and $R_{1} \rightarrow 1.31$ for $p=3.3$. Since the differences are too small, we do not show the counterparts of Figs. 3 and 4 for $p=2.3$ and 3.3.

\section{SUMMARY AND DISCUSSION}

We have derived the $\beta$-dependence on $a$ both in the cooling-limited and escape-limited cases although based on the test-particle approximation for stationary shocks. We claim that the cutoff shape of the particle distribution and/or radiation spectrum potentially becomes a new tool to study the acceleration 


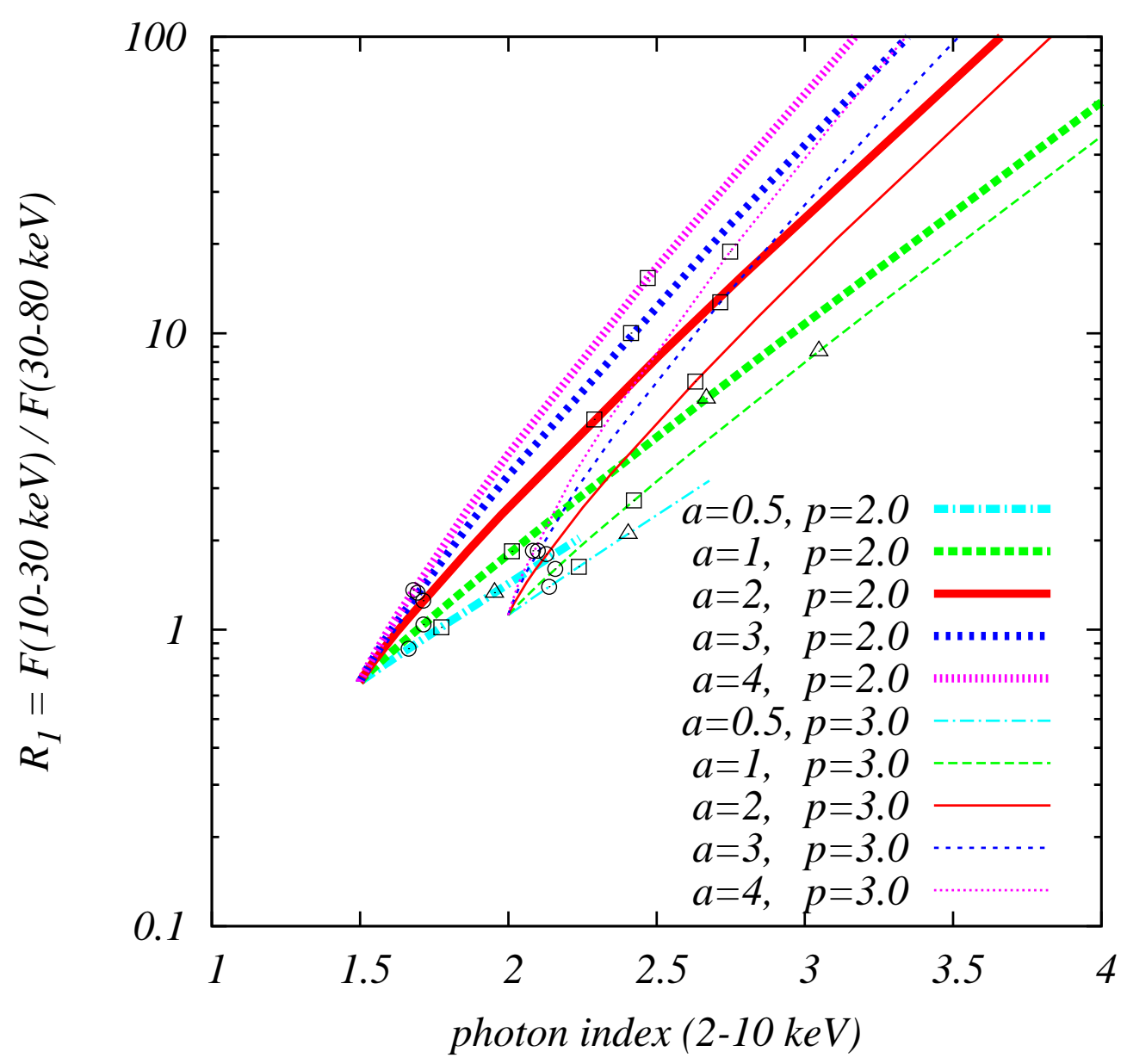

Fig. 2 The flux ratio $R_{1}=F(10-30 \mathrm{keV}) / F(30-80 \mathrm{keV})$ as a function of the $2-10 \mathrm{keV}$ photon index. The meanings of each line and point are the same as in Fig. 1.

mechanism. Then, we have calculated the spectral properties of synchrotron radiation for an electron distribution given by Equation (1) in terms of the flux ratios and photon indices in various energy bands. We have presented typical reference lines predicted by the simplest one-zone, synchrotron model. All the figures in our paper can be directly compared with observations in the near future. In the next era of hard X-ray observation with $N U S T A R$ and ASTRO-H, the observed data should first be compared with those lines. If the data deviate from the prediction of the model, it will provide us information on the acceleration mechanism, radiation mechanism, the inhomogeneity of the source (validity of one-zone model), and so on. Note that if we consider the softer band (2-10 keV) in addition to the harder bands $(>10 \mathrm{keV})$, then the energy range becomes broader, so that we can easily constrain spectral parameters.

The value of $p$ may be determined by the slope of the radio synchrotron and/or GeV-to-TeV gammaray spectrum. Once $p$ is independently determined, then comparing theoretical lines of Figs. 1, 2, 5, 0 with observed data, which will be provided by NUSTAR and/or ASTRO-H, we can constrain the value of a. As discussed in Section 2.2, it is helpful if the acceleration is age-limited, cooling-limited or escape- 


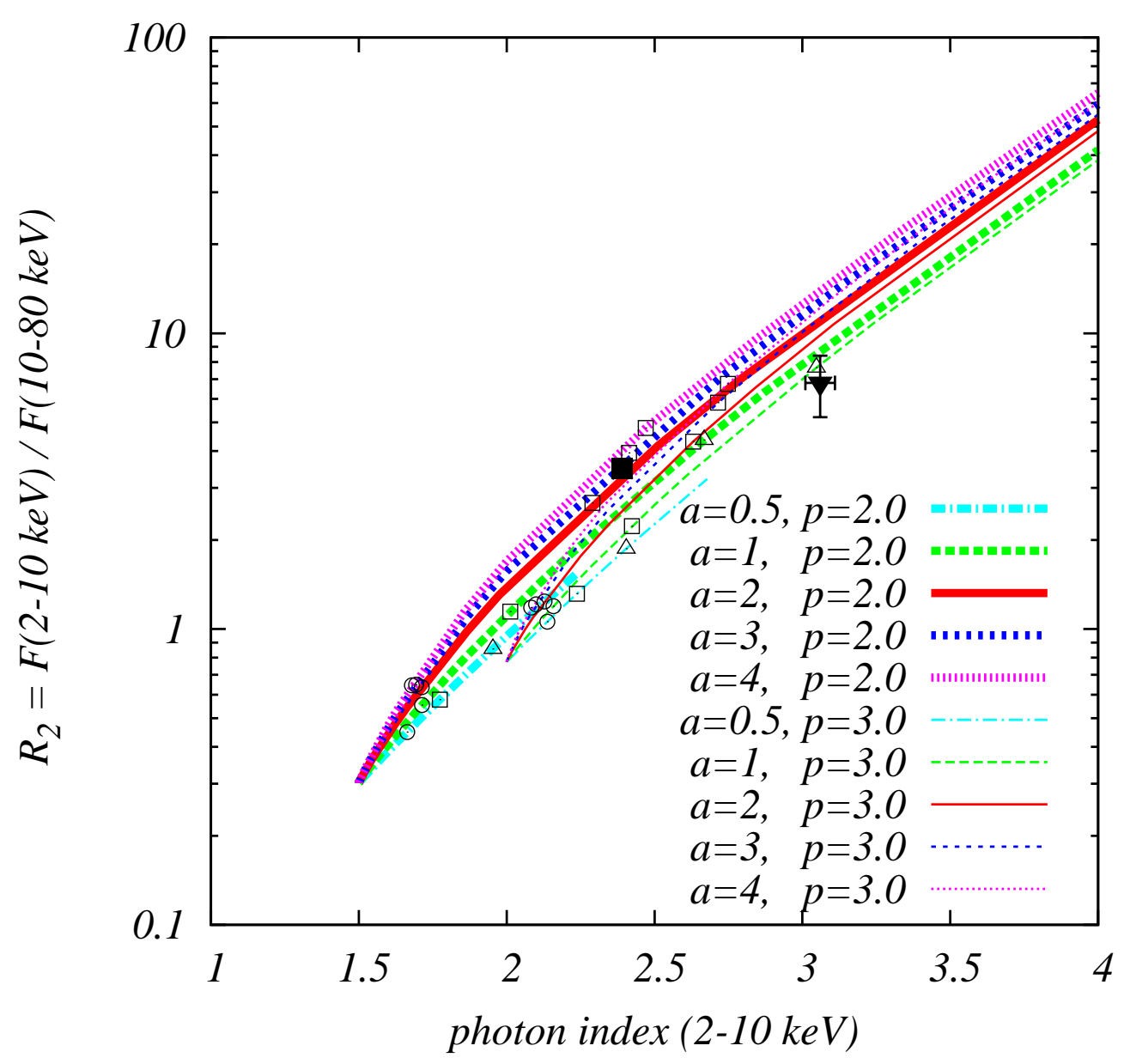

Fig. 3 The flux ratio $R_{2}=F(2-10 \mathrm{keV}) / F(10-80 \mathrm{keV})$ as a function of the $2-10 \mathrm{keV}$ photon index. The meanings of each line and point are the same as in Figure 1.

limited. In particular, we can check if $a \approx 2$ or not, which corresponds to the popular case of Bohm diffusion in age- or cooling-limited acceleration. One may expect that $a=2$ is typical. Indeed, some observational results are consistent with the case of $a=2$ (e.g., Nakamura et al.2012), although no firm conclusion has been derived.

In addition to other observational information such as a wide-band radiation spectrum, spatial and temporal variability in X-ray and so on, the value of $a$ will provide another independent constraint on the magnetic field strength (for a typical example, see Section 4.1.1), which, however, is not always applicable. From another point of view, if we know, by other observations, the field strength and understand how $E_{\max , \mathrm{e}}$ is determined, then with the aid of Eq. (3), (4) or (5), we can further constrain the value of $\beta$, which gives us rich information on the acceleration process, in particular, the electron transport mechanism.

Note that our present method is able to constrain the value of a without detailed spectral fitting which needs brightness. It is easier than the spectral fitting analysis to observationally determine the 


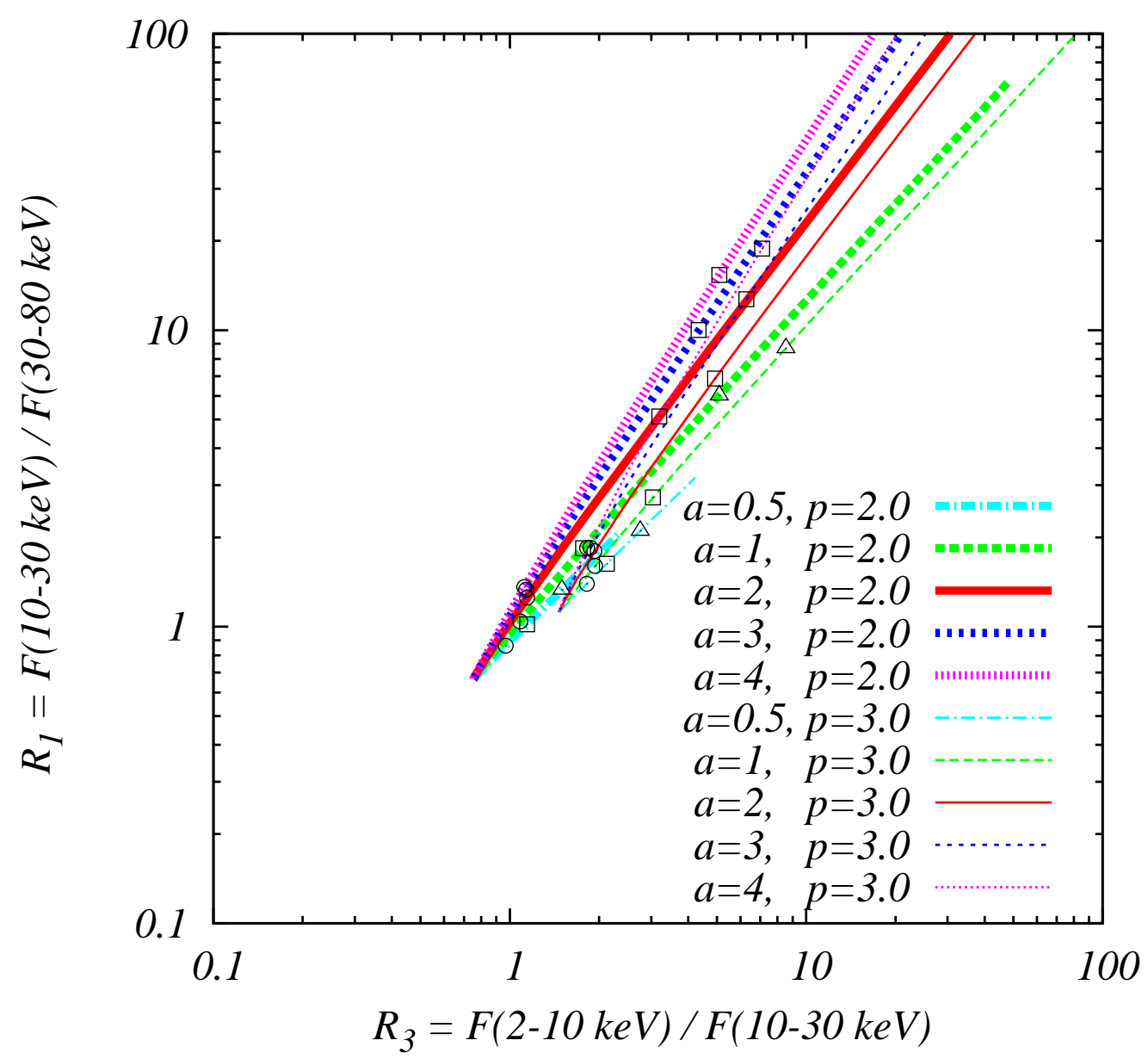

Fig. 4 The flux ratio $R_{1}=F(10-30 \mathrm{keV}) / F(30-80 \mathrm{keV})$ as a function of $R_{3}=F(2-$ $10 \mathrm{keV}) / F(10-30 \mathrm{keV})$. The meanings of each line and point are the same as in Figure 1.

flux ratios and/or photon index. Even for sources whose X-ray brightness is too small to perform a precise spectral fitting, we will be able to discuss the spectral parameter $a$. Hence we can obtain a larger sample, which enables us to do the statistical analysis. Consequently, we will be able to extract more general properties of particle acceleration at SNRs, which cannot be done by individual analysis for the small number of bright sources.

Figure 3 shows that all the curves are degenerate with each other, along which the observed data points should lie if the X-ray emission arises from synchrotron radiation. If there are outliers, we can discuss the existence of extra components, such as a bump in the electron spectrum near $E_{\max , \mathrm{e}}$ due to effects of pile-up via synchrotron cooling (Longair 1994, Drury et al. 1999, Zirakashvili \& Aharonian 2007) and/or nonlinear acceleration (e.g., Malkov \& Drury 2001), jitter or diffusive synchrotron emission (Toptygin \& Fleishman 1987; Medvedev 2000, Reville \& Kirk 2010; Teraki \& Takahara 2011), secondary synchrotron radiation that originates from accelerated protons generating charged pions (e.g., Yamazaki et al.2006), nonthermal bremsstrahlung emission (Laming 2001a, 2001b, 


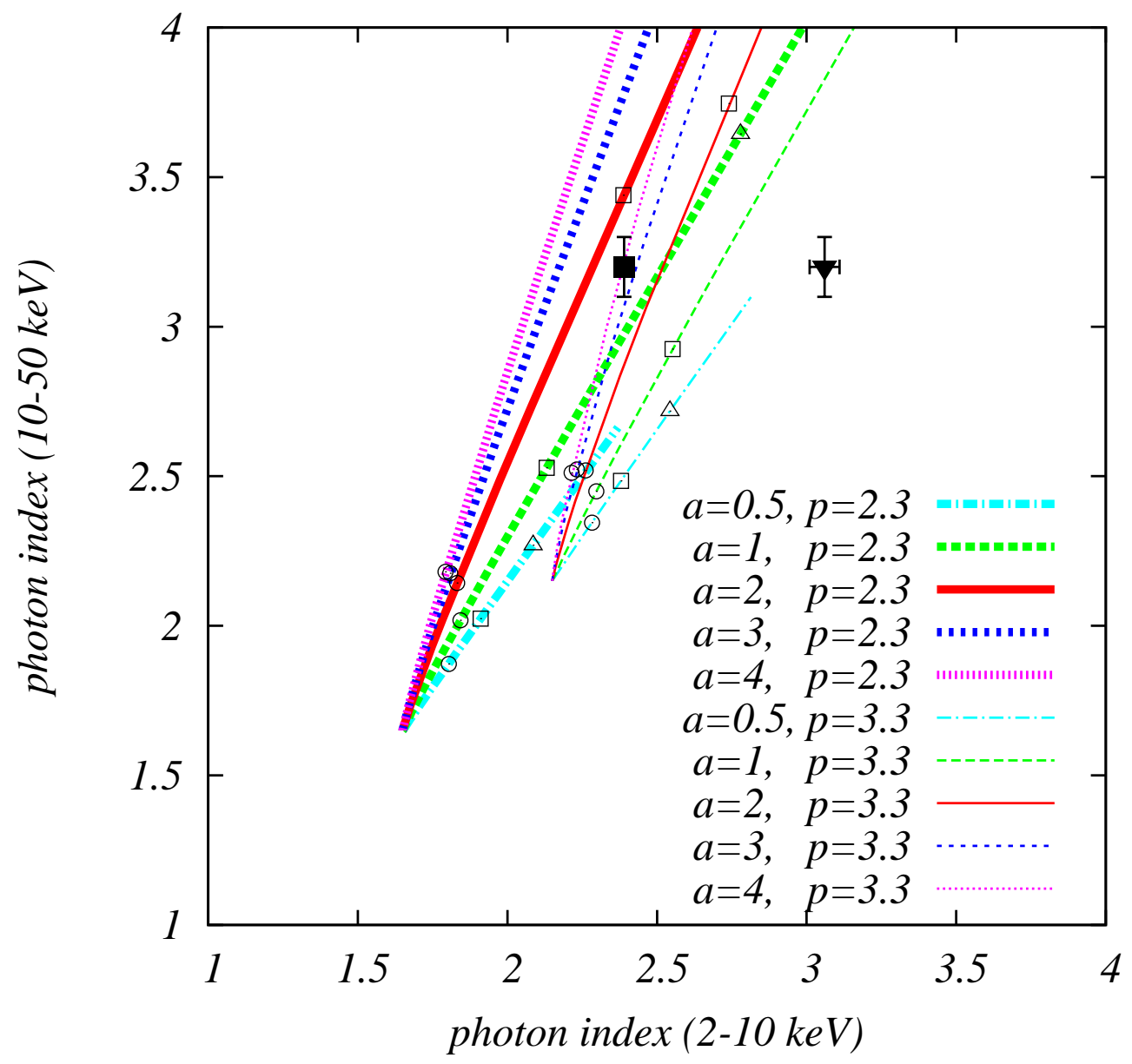

Fig. 5 The same as in Fig. 1 but for $p=2.3$ and 3.3.

Vink \& Laming 2003, Vink 2008, Ohira et al.2012a), and so on. Similar discussion may be done with Fig. 4 .

A caveat is that the one-zone model is adopted for simplicity. Clearly our plots change if we see emissions from different regions which have a different parameter set of $B, E_{\max , \mathrm{e}}, p, a$ as well as flux normalization. In order to avoid or minimize this problem, hard X-ray observations of small emission regions are necessary. In this sense, the hard X-ray imaging system onboard NuSTAR or ASTRO-H is useful.

In this paper, we have focused on hard X-rays which arise from synchrotron radiation of electrons near $E_{\max , \mathrm{e}}$. It may be possible to do a similar analysis in gamma-ray bands using next generation gamma-ray telescopes like CTA (Actis et al.2011). However, at present there are several uncertainties. First, the emission mechanism is uncertain: even for the representative case of SNR RX J1713.7-3946, we have not yet determined whether the gamma-ray emission is leptonic or hadronic (see Section 4.1.1). Second, in the leptonic case, where the gamma-rays arise from inverse Compton emission of accelerated electrons, seed photons are uncertain; in addition to CMB, infrared and optical photons, whose intensity 


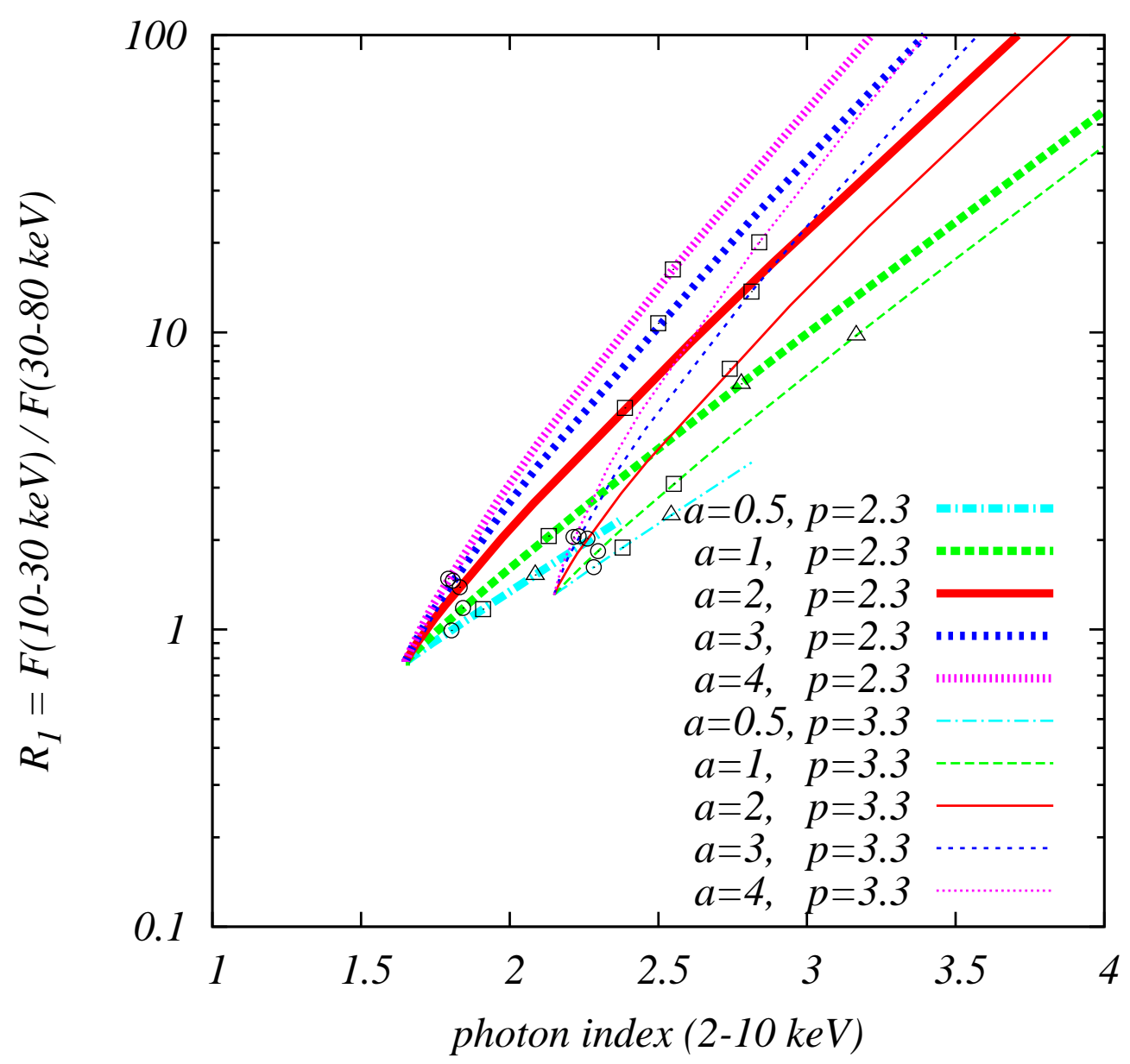

Fig. 6 The same as in Fig. 2 but for $p=2.3$ and 3.3.

is uncertain and depends on the position of SNRs in the Galactic plane, significantly contribute to the gamma-ray emission. Finally, systematic error of the gamma-ray measurement might be too large for our discussion. If all of the above uncertainties are removed, the flux ratio and/or photon index in gamma-ray bands will be another independent diagnostic. In particular, if gamma-rays are hadronic, we can obtain the cutoff shape of protons near $E_{\max , p}$, which cannot be done by hard X-ray analysis. However, even in this case, we need to do a careful analysis because spatial inhomogeneity of target matter modifies the gamma-ray spectrum (e.g., Ohira et al.2011; Inoue et al.2012).

\subsection{Application to specific cases}

In the following, let us consider the case of Cas A and RX J1713.7-3946 as representative examples. 


\subsubsection{RX J1713.7-3946}

Tanaka et al. (2008) presented results from Suzaku observations of RX J1713.7-3946. XIS and HXD, onboard Suzaku, detected X-rays from this SNR in $0.4-12 \mathrm{keV}$ and $12-40 \mathrm{keV}$ bands, and measured photon indices in these bands to be $2.39 \pm 0.01$ and $3.2 \pm 0.1$, respectively. We adopt these values as 2-10 keV and 10-50 keV photon indices, respectively. Furthermore, using the results of spectral analysis from Tanaka et al. (2008), we calculate the energy flux of the whole SNR as $F(2-10 \mathrm{keV})=$ $(4.59 \pm 0.04) \times 10^{-10} \mathrm{erg} \mathrm{s}^{-1} \mathrm{~cm}^{-2}$ and $F(10-80 \mathrm{keV})=(1.32 \pm 0.05) \times 10^{-10} \mathrm{erg} \mathrm{s}^{-1} \mathrm{~cm}^{-2}$, which lead to the flux ratio, $R_{2}=3.5 \pm 0.2$. The derived value of $R_{2}$ and the adopted $2-10 \mathrm{keV}$ photon index are on theoretical lines in Fig. 3, which implies that the X-ray emission is the synchrotron radiation.

Recently Fermi measured the gamma-ray spectrum of RX J1713.7-3946 in the 3-300 GeV energy range, whose photon index $(1.5 \pm 0.1)$ is typical for leptonic inverse Compton emission from highenergy electrons with the spectral index $p \approx 2.0$ (Abdo et al.2011). Hence, at first, let us consider the possibility that gamma-rays are emitted by inverse Compton scattering. Assuming CMB and infrared photons are the seeds of the scattering, $\mathrm{Li}$ et al. (2011) extracted the electron distribution from the observed gamma-ray spectrum and obtained $p \approx 2.0$ and $a \approx 0.6$, although the uncertainty is large. Lee et al. (2012) set $a=0.5$ to explain the gamma-ray spectrum. The value $a \approx 0.6$ implies $\beta=0.3$ in the age-limited case, but $\beta=-0.4$ in the cooling-limited case. The latter is unlikely because the negative value of $\beta$ is quite unnatural in the context of magnetic turbulence. This is also inferred from the fact that the flux ratio of synchrotron X-rays to inverse Compton gamma-rays leads to the field strength $B \sim 10-20 \mu \mathrm{G}$ (e.g., Katz \& Waxman 2008, Yamazaki et al. 2009, Ellison et al. 2010), so that the effect of synchrotron cooling is not significan[2. However, one can find from Figs. 1 and 5 that no lines for $a=0.5$ are consistent with the measured photon indices in the $2-10 \mathrm{keV}$ and $10-50 \mathrm{keV}$ bands. In particular, $a \approx 1.5$ if $p \approx 2.0$. This fact was indicated by Tanaka et al. (2008), in which they found that the X-ray spectrum taken by Suzaku is consistent with both cases $a=1$ and $a=2$. Therefore, the simple leptonic inverse Compton model fails to simultaneously explain both the X-ray and the gamma-ray spectral shapes.

On the other hand, hadronic scenarios for the observed hard gamma-ray spectrum are still viable if we consider the shock-cloud interaction (Inoue et al.2012) or extreme limit of nonlinear particle acceleration (e.g., Yamazaki et al.2009). In these cases, a strong magnetic field ( $B \sim 0.1-1 \mathrm{mG})$ is predicted, which is also inferred from the detection of time variability and thin filaments of the synchrotron X-rays, although there are some counter arguments (e.g., Katz \& Waxman2008, Bykov et al. 2008, Reynolds et al. 2012). Hence, the electron acceleration is limited by synchrotron cooling, so that the spectral index of electrons near $E_{\max , \mathrm{e}}$ should be $p \geq 3.0$ (see Section 2.1 . One can see from Fig. 1 that if $p \approx 3.0$, then the observed photon indices in the $2-10 \mathrm{keV}$ and $10-50 \mathrm{keV}$ bands tell us $a \geq 2$, so that $\beta \geq 1$. Therefore, Bohm diffusion is consistent with observations.

Note that, however, this argument is not conclusive. We consider the spectrum of the whole SNR. As discussed previously, a one-zone model may not be adequate. Further observations resolving smaller emission regions are necessary.

\subsubsection{Cas A}

XIS and HXD onboard Suzaku have measured the spectrum in the 3.4-40 keV band (Maeda et al. 2009). They found that the non-thermal component in this energy range is described by a single power-law form with a photon index of $3.06 \pm 0.05$. Here we adopt this value as the $2-10 \mathrm{keV}$ photon index, although a thermal component prevents us from precisely determining the index. Using their fitting parameter, we have $F(2-10 \mathrm{keV})=(6.5 \pm 0.9) \times 10^{-10} \mathrm{erg} \mathrm{s}^{-1} \mathrm{~cm}^{-2}$. Other observations in higher energy bands such as Swift/BAT (14-195 keV) (Baumgartner et al. 2012) and BeppoSAX/PDS (15$300 \mathrm{keV}$ ) (Vink \& Laming 2003), show a softer photon index of 3.26 \pm 0.09 and $3.32 \pm 0.05$, respectively. Hence we expect the photon index in the $10-50 \mathrm{keV}$ band is slightly softer (3.1-3.3) than the value

2 There are several possibilities to explain the observed time variability and thin filaments of the synchrotron X-rays without amplification of the magnetic field (e.g., Katz \& Waxman 2008, Bykov et al.2008. 
3.06 measured by Suzaku. Using the parameters given by Swift/BAT, we have $F(10-80 \mathrm{keV})=$ $(9.6 \pm 0.7) \times 10^{-11} \mathrm{erg} \mathrm{s}^{-1} \mathrm{~cm}^{-2}$. Then we obtain the flux ratio $R_{2} \approx 6.8 \pm 1.6$.

Although the index of the electron spectrum, $p$, is not yet fixed, the measured gamma-ray spectrum shows that $p$ is larger than 2.0 (Abdo et al. 2010). Here we adopt $p \approx 2.3$ as a typical value. Then, one can plot the observed data in Figures 3 and 5 . Placing the observed 2-10 keV photon index of $3.06 \pm 0.05$ and the flux ratio $R_{2} \approx 6.8 \pm 1.6 \mathrm{in} \mathrm{Fig.} \mathrm{3,} \mathrm{we} \mathrm{find} \mathrm{that} \mathrm{the} \mathrm{data} \mathrm{are} \mathrm{marginally} \mathrm{consistent}$ with the theoretical prediction for the synchrotron radiation. However, the observed data point of 2$10 \mathrm{keV}$ and 10-50 keV photon indices does not lie on any lines in Fig. 5. Furthermore, the observed data show that for a given value of 2-10 keV photon index, the 10-50 keV photon index is smaller than expected via the synchrotron radiation. Hence, one can claim the existence of an extra component. At present, the origin of the hard X-rays above $10 \mathrm{keV}$ for Cas $\mathrm{A}$ is a matter of debate. In particular we are interested in whether the emission is nonthermal bremsstrahlung or synchrotron (Laming 2001a, 2001b, Vink \& Laming 2003, Vink 2008). Our discussion here may contribute to this topic. Note that, however, this argument is based on a one-zone model. At present, it is uncertain whether $2-10 \mathrm{keV}$ and 10-80 keV X-rays come from the same emission region, which is left for future observations.

One may insist on explaining the observed photon indices by only the synchrotron radiation. Indeed, it is marginally possible that the observed 2-10 keV and 10-50 keV photon indices lie on the theoretical line of $p \geq 3$ and $a \approx 0.5$. In this case, the acceleration is not limited by synchrotron cooling because generally $\beta>0$ is expected (see Eq. 3). This is apparently inconsistent with the observational implications that magnetic field strength is large enough $(B \geq 0.5 \mathrm{mG})$ for the electron acceleration to be cooling limited (Vink \& Laming 2003, Bamba et al.2005a).

Acknowledgements We would like to thank Tsunehiko Kato, Kohta Murase, Takanori Sakamoto, Atsumasa Yoshida, Tsuyoshi Inoue and Tohru Shibata, and the anonymous referee for useful comments. This work was supported in part by the fund from Research Institute, Aoyama Gakuin University (R. Y. and A. B.), and by grant-in-aid from the Ministry of Education, Culture, Sports, Science, and Technology (MEXT) of Japan, No. 24.8344 (Y. O.), No. 24840036 (M. S.) and No. 22684012 (A. B.)

\section{Appendix A: CHARACTERISTIC ENERGIES}

In this section, we assume the Bohm diffusion, $K(E) \propto E$, for simplicity. More detailed analysis is found in Ohira et al. (2012b). The extension to a more general case $\left(K(E) \propto E^{\beta}\right)$ is easy and omitted here.

\section{A.1. Maximum energy of accelerated electrons and protons}

Let $v_{\mathrm{s}}$ and $B_{\mathrm{d}}$ be the shock velocity and the downstream magnetic field, respectively. First, suppose that the maximum energy of electrons is determined from the balance of the synchrotron loss and acceleration. Then, equating the acceleration time $t_{\mathrm{acc}}(E)=20 \xi c E / 3 e v_{\mathrm{s}}^{2} B_{\mathrm{d}}$ with the synchrotron cooling time, $t_{\text {syn }}(E)=125 \mathrm{yr}(E / 10 \mathrm{TeV})^{-1}\left(B_{\mathrm{d}} / 100 \mu \mathrm{G}\right)^{-2}$, we obtain

$$
E_{\max }^{(\operatorname{cool})}=\frac{24}{\xi^{1 / 2}}\left(\frac{v_{\mathrm{s}}}{10^{8} \mathrm{~cm} \mathrm{~s}^{-1}}\right)\left(\frac{B_{\mathrm{d}}}{10 \mu \mathrm{G}}\right)^{-1 / 2} \mathrm{TeV}
$$

where $\xi$ is a gyro factor. On the other hand, if the cooling is not significant, that is, $t_{\text {acc }}(E), t_{\text {age }} \ll$ $t_{\mathrm{syn}}(E)$, then the maximum energy can be determined by the condition $t_{\mathrm{acc}}(E)=t_{\text {age }}$, and we obtain

$$
E_{\max }^{(\text {age })}=\frac{4.8 \times 10^{2}}{\xi}\left(\frac{v_{\mathrm{s}}}{10^{9} \mathrm{~cm} \mathrm{~s}^{-1}}\right)^{2}\left(\frac{B_{\mathrm{d}}}{10 \mu \mathrm{G}}\right)\left(\frac{t_{\text {age }}}{10^{3} \mathrm{yr}}\right) \mathrm{TeV} .
$$

For young SNRs, we typically expect the maximum energies of electrons and protons, $E_{\max , \mathrm{e}}$ and $E_{\max , \mathrm{p}}$ are $E_{\max }^{(\mathrm{cool})}$ and $E_{\max }^{(\text {age }}$, respectively. Then, using equation (A.1) and (A.2), we eliminate $v_{\mathrm{s}}^{2} / \xi$ 
and obtain

$$
E_{\mathrm{max}, \mathrm{p}} \approx 83\left(\frac{E_{\mathrm{max}, \mathrm{e}}}{10 \mathrm{TeV}}\right)^{2}\left(\frac{B_{\mathrm{d}}}{100 \mu \mathrm{G}}\right)^{2}\left(\frac{t_{\mathrm{age}}}{10^{3} \mathrm{yr}}\right) \mathrm{TeV}
$$

\section{A.2. The cooling break energy}

Let $t$ be the characteristic time of SNR evolution, which may be the expansion time of $t_{\text {age }}$ itself. Then the cooling break, $E_{\mathrm{b}}$, appears in the electron spectrum at the energy where $t_{\mathrm{syn}}\left(E_{\mathrm{b}}\right)=t$, that is,

$$
E_{\mathrm{b}}=12.5 \mathrm{TeV}\left(\frac{t}{10^{2} \mathrm{yr}}\right)^{-1}\left(\frac{B_{\mathrm{d}}}{100 \mu \mathrm{G}}\right)^{-2} .
$$

The cooling break appears if $E_{\mathrm{b}}<E_{\max , \mathrm{e}}=E_{\max }^{(\mathrm{cool})}$, which can be rewritten as

$$
B_{\mathrm{d}}>139 \mu \mathrm{G} \xi^{1 / 3}\left(\frac{v_{\mathrm{s}}}{10^{8} \mathrm{~cm} \mathrm{~s}^{-1}}\right)^{-2 / 3}\left(\frac{t}{10^{2} \mathrm{yr}}\right)^{-2 / 3} .
$$

\section{Appendix B: ANALYTICAL DERIVATION OF ASYMPTOTIC SPECTRAL SHAPE}

\section{B.1. The case of cooling-limited acceleration}

Zirakashvili \& Aharonian (2007) obtained the asymptotic electron spectrum near the maximum electron energy in the cooling-dominated phase, such as

$$
\begin{gathered}
N(E)=A_{0}(E) \exp \left[S_{0}(E)\right], \\
A_{0}(E)=E^{-1 / 2} \exp \left[\int^{E} \frac{\sqrt{K_{2}} \frac{\partial}{\partial E} \sqrt{b_{2}}+\sqrt{K_{1}} \frac{\partial}{\partial E} \sqrt{b_{1}}}{\sqrt{b_{1} K_{1}}+\sqrt{b_{2} K_{2}}} d E^{\prime}\right], \\
S_{0}(E)=-\left(\frac{\gamma_{s}}{v_{\mathrm{s}}}\right)^{2} \int^{E}\left(\frac{\sqrt{b_{1} K_{1}}+\sqrt{b_{2} K_{2}}}{E^{\prime}}\right)^{2} d E^{\prime},
\end{gathered}
$$

where $\gamma_{s}=3 r /(r-1)\left(r\right.$ is the shock compression ratio), and $v_{\mathrm{s}}$ is the shock velocity. Functions $K(E)$ and $b(E)=d E / d t$ are the diffusion coefficient and energy loss rate of electrons, respectively. Subscripts 1 and 2 indicate upstream and downstream regions of the shock, respectively. In the case of synchrotron cooling, $b(E)$ is proportional to $E^{2}$.

We assume that $K(E)=K_{0} E^{\beta}$ and $b(E)=b_{0} E^{2}$ where $K_{0}$ and $b_{0}$ are constants, and that ratios $b_{1} / b_{2}$ and $K_{1} / K_{2}$ are also constant. Then we obtain

$$
A_{0}(E) \propto E^{1 / 2} .
$$

However, for typical parameters of young SNRs, this positive slope can hardly be seen in the X-ray emission. Furthermore, neglecting non-dimensional terms on the order of unity, we derive $S_{0}(E) \approx$ $-\left(K_{0} b_{0} / v_{\mathrm{s}}^{2}\right) E^{\beta+1}$. By the way, acceleration time and synchrotron cooling time are calculated as $t_{\text {acc }}(E) \approx K_{0} E^{\beta} / v_{\mathrm{s}}^{2}$ and $t_{\mathrm{syn}}(E) \approx\left(b_{0} E\right)^{-1}$, respectively, where we again neglect terms on the order of unity. Then equating them, we obtain $E_{\max }^{(\mathrm{cool})} \approx\left(v_{\mathrm{s}}^{2} / K_{0} b_{0}\right)^{1 /(\beta+1)}$. Therefore, we finally derive

$$
S_{0}(E) \approx-\left(\frac{E}{E_{\max }^{(\text {cool })}}\right)^{\beta+1}
$$




\section{B.2. The case of escape-limited acceleration}

We assume the test-particle regime and place a free escape boundary upstream at a distance of $\ell$ away from the shock front $x=0$, that is, the particle distribution function is zero at $x=-\ell(<0)$. Then the stationary transport equation is solved to find a particle spectrum around the shock front given by (Caprioli et al.2009, Reville et al.2009)

$$
N(E) \propto \exp \left\{-\frac{3 r}{r-1} \int^{E} \frac{\mathrm{d} \log E^{\prime}}{1-\exp \left[-v_{\mathrm{s}} \ell / K\left(E^{\prime}\right)\right]}\right\},
$$

where $v_{\mathrm{s}}$ is the velocity of the shock. In the escape-limited case, the maximum energy is determined by the condition (Ohira et al.2010)

$$
\frac{K\left(E_{\max }^{(\mathrm{esc})}\right)}{v_{\mathrm{s}}}=\ell .
$$

Then, one can see $K(E) / v_{\mathrm{s}} \ell=K(E) / K\left(E_{\max }^{(\mathrm{esc})}\right)=\left(E / E_{\max }^{(\mathrm{esc})}\right)^{\beta}$. Changing variables into $y\left(E^{\prime}\right)=$ $\left(E^{\prime} / E_{\max }^{(\text {esc })}\right)^{\beta}$, we derive

$$
N(E) \propto \exp \left\{-\frac{3 r}{r-1} \times \frac{1}{\beta} \int^{y(E)} \frac{\mathrm{d} \log y}{1-e^{-1 / y}}\right\} .
$$

In the case of $E \ll E_{\max }^{(\mathrm{esc})}$, the term $e^{-1 / y}$ can be neglected, so that we obtain $N(E) \propto E^{-3 r /(r-1)}$. On the other hand, if $E \gg E_{\max }^{(\text {esc })}$, we approximate $1-e^{-1 / y} \approx 1 / y$, resulting in $N(E) \propto$ $\exp \left[-\left(E / E_{\max }^{(\mathrm{esc})}\right)^{\beta}\right]$, where a term on the order of unity is again neglected.

\section{References}

Abdo, A. A. et al. 2010, ApJ, 710, L92

Abdo, A. A. et al. 2011, ApJ, 734, 28

Actis, M. et al. 2011, Exp. Astron., 32, 193

Bamba, A. et al. 2003, ApJ, 589, 827

Bamba, A. et al. 2005a, ApJ, 621, 793

Bamba, A. et al. 2005b, ApJ, 632, 294

Baumgartner, W. H. et al. 2013, ApJS, 207, 19

Bell, A. R. 1978, MNRAS, 182, 147

Blandford, R. D., \& Ostriker, J. P., 1978, ApJ, 221, L29

Blandford, R. D., \& Eichler, D., 1987, Phys. Rep., 154,1

Bykov, A. M. et al. 2000, ApJ, 538, 203

Bykov, A. M., Uvarov, Y. A., \& Ellison, D. C. 2008, ApJ, 689, L133

Caprioli et al. 2009, MNRAS, 396, 2065

Drury, L. O’ C., Duffy, P., Eichler, D., \& Mastichiadis, A. 1999, A\&A, 347, 370

Drury, L. O’ C., Aharonian, F. A., Malyshev, D., \& Gabici, S. 2009, A\&A, 496, 1

Ellison, D. C. \& Bykov, A. M. 2011, ApJ, 731, 87

Ellison, D. C. et al. 2010, ApJ, 712, 287

Giordano, F. et al. 2012, ApJ, 744, L2

Hailey C. J. et al. 2010, in Society of Photo-Optical Instrumentation Engineers (SPIE) Conference Series, 7732, $77320 \mathrm{~T}$

Harrison, F. A. et al. 2013, ApJ, 770, 103 
Inoue, T. et al. 2012, ApJ, 744, 71

Kang, H., Ryu, D., \& Jones, T. W. 2009, ApJ, 695, 1273

Kato, T. \& Takahara, F. 2003, MNRAS, 342, 639

Katz, B., \& Waxman, E. 2008, J. Cosmology Astropart. Phys., 01, 018

Koyama, K. et al. 1995, Nature, 378, 255

Laming, J. M. 2001a, ApJ, 546, 1149

Laming, J. M. 2001b, ApJ, 563, 828

Lee, S.-H. et al. 2012, ApJ, 750, 156

Li, H, Liu, S., \& Chen, Y. 2011, ApJ, 742, L10

Longair, M. S. 1994, High Energy Astrophysics, Vol. 2, Stars, the Galaxy and the Interstellar Medium (Cambridge: Cambridge Univ. Press)

Maeda, Y. et al. 2009, PASJ, 61, 1217

Malkov, M. A., \& Drury, L. O’ C. 2001, Rep. Prog. Phys., 64, 429

Medvedev, M. V. 2000, ApJ, 540, 704

Nakamura, R. et al. 2012, ApJ, 746, 134

Ohira, Y. et al. 2010, A\&A, 513, A17

Ohira, Y. et al. 2011, MNRAS, 410, 1577

Ohira, Y. et al. 2012a, MNRAS, 421, L102

Ohira, Y. et al. 2012b, MNRAS, 427, 91

Ptuskin, V. S., \& Zirakashvili, V. N. 2005, A\&A, 429, 755

Putze, A., Derome, L., Maurin, D., Perotto, L., \& Taillet, R. 2009, A\&A, 497, 991

Reville, B., Kirk, J. G., \& Duffy, P. 2009, ApJ, 694, 951

Reville, B. \& Kirk, J. G. 2010, ApJ, 724, 1283

Reynolds, S. P. 1998, ApJ, 493, 375

Reynolds, S. P., \& Keohane, J. W. 1999, ApJ, 525, 368

Reynolds, S. P., Gaensler, B. M., \& Bocchino, F. 2012, Space Science Reviews, 166, 231

Shibata, T., Ishikawa, T., \& Sekiguchi, S. 2011, ApJ, 727, 38

Strong, A. W., \& Moskalenko, I. V. 1998, ApJ, 509, 212

Tanaka, T. et al. 2008, ApJ, 685, 988

Takahashi, T. et al. 2010, in Society of Photo-Optical Instrumentation Engineers (SPIE) Conference Series, 7732, $77320 Z$

Teraki, Y. \& Takahara, F. 2011, ApJ, 735, L44

Toptygin, I. N., \& Fleishman, G. D. 1987, Ap\&SS. 132, 213

Uchiyama, Y. et al. 2007, Nature, 449, 576

Uchiyama, Y. \& Aharonian, F. A. 2008, ApJ, 677, L105

Vink, J. 2008, A\&A, 486, 873

Vink, J. \& Laming, J. M. 2003, ApJ, 584, 758

Yamazaki, R. et al. 2004, A\&A, 416, 595

Yamazaki, R. et al. 2006, MNRAS, 371, 1975

Yamazaki, R., Kohri, K., \& Katagiri, H. 2009, A\&A, 495, 9

Zirakashvili, V. N. \& Aharonian, F. 2007, A\&A, 465, 695

This paper was prepared with the RAA LATEX macro v1.2. 